\title{
Downregulation of miR-486-5p in papillary thyroid carcinoma tissue: A study based on microarray and miRNA sequencing
}

\author{
DONG-YUE WEN $^{1}$, DENG-HUA PAN ${ }^{1}$, PENG LIN $^{1}$, QIU-YAN MO ${ }^{1}$, YUN-PENG WEI ${ }^{1}$, \\ YI-HUAN LUO ${ }^{2}$, GANG CHEN $^{3}$, YUN HE ${ }^{1}$, JUN-QIANG CHEN ${ }^{2}$ and HONG YANG ${ }^{1}$ \\ Departments of ${ }^{1}$ Medical Ultrasonics, ${ }^{2}$ Gastrointestinal Surgery and ${ }^{3}$ Pathology, First Affiliated Hospital of \\ Guangxi Medical University, Nanning, Guangxi Zhuang Autonomous Region 530021, P.R. China
}

Received November 9, 2017; Accepted May 17, 2018

DOI: $10.3892 / \mathrm{mmr} .2018 .9247$

\begin{abstract}
Abnormal expression of microRNA (miR) is associated with the occurrence and progression of various types of cancers, including papillary thyroid carcinoma (PTC). In the present study, the aim was to explore miR-486-5p expression and its role in PTC, as well as to investigate the biological function of its potential target genes. The expression levels of miR-486-5p and its clinicopathological significance were examined in 507 PTC and 59 normal thyroid samples via The Cancer Genome Atlas (TCGA). Subsequently, the results were validated using data from Gene Expression Omnibus (GEO) and ArrayExpress. Receiver operating characteristic and summary receiver operating characteristic curves were used to assess the ability of miR-486-5p in distinguishing PTC from normal tissue. Furthermore, potential miR-486-5p mRNA targets were identified using 12 prediction tools and enrichment analysis was performed on the encoding genes using Gene Ontology and Kyoto Encyclopedia of Genes and Genomes. The expression levels of miR-486-5p were consistently downregulated in PTC compared with in normal tissue across datasets from TCGA, GEO (GSE40807, GSE62054 and GSE73182) and ArrayExpress (E-MTAB-736). The results also demonstrated that miR-486-5p expression was associated with cancer stage $(\mathrm{P}=0.003)$, pathologic lymph node $(\mathrm{P}=0.047)$, metastasis $(\mathrm{P}=0.042)$, neoplasm $(\mathrm{P}=0.012)$ and recurrence $(\mathrm{P}=0.016)$ in patients with PTC. In addition, low expression of miR-486-5p in patients with
\end{abstract}

Correspondence to: Professor Hong Yang, Department of Medical Ultrasonics, First Affiliated Hospital of Guangxi Medical University, 6 Shuangyong Road, Nanning, Guangxi Zhuang Autonomous Region 530021, P.R. China

E-mail: yanghonggx@163.com

Abbreviations: PTC, papillary thyroid carcinoma; TCGA, the cancer genome atlas; GEO, gene expression omnibus; ROC, receiver operating characteristic; SROC, summary receiver operating characteristic; OS, overall survival; GO, gene ontology; KEGG, Kyoto encyclopedia of genes and genomes, PPI, protein-protein interaction

Key words: miR-486-5p, papillary thyroid carcinoma, bioinformatics analysis, hub genes
PTC was associated with a worse overall survival. A total of $80 \mathrm{miR}-486-5 \mathrm{p}$-related genes were observed from at least 9 of 12 prediction platforms, and these were involved in 'hsa05200: Pathways in cancer' and 'hsa05206: MicroRNAs in cancer'. Finally, three hub genes, CRK like proto-oncogene, phosphatase and tensin homolog and tropomyosin 3, were identified as important candidates in tumorigenesis and progression of PTC. In conclusion, it may be hypothesized that miR-486-5p contributes towards PTC onset and progression, and may act as a clinical target. However, in vitro and in vivo experiments are required to validate the findings of the present study.

\section{Introduction}

MicroRNAs (miRNAs/miRs) consist of a large family of non-coding, single-stranded RNA molecules that are 18-22 nucleotides long. These RNA molecules modulate gene expression post-transcriptionally by regulating the translation and stability of mRNAs. This regulation is achieved through complete or incomplete sequence matching between miRNAs and the 3'untranslated regions of the target mRNA (1). So far, thousands of mature miRNAs have been identified, of which $\sim 2,600$ human miRNAs have been annotated in the miRbase database (http://www.mirbase.org/).

The incidence of papillary thyroid carcinoma (PTC) continues to increase worldwide (2), and it has been demonstrated that miRNAs may contribute to occurrence and progression of the disease. Differential miRNA expression has been reported in PTC vs. normal tissue samples (3-5); certain miRNAs, including serum miR-34a, -155, -197, -221, -224 and -375, can be used as diagnostic and prognostic indicators of thyroid cancer (6,7). The mechanism of action for miR-150, -422a, -18a and -19a in human thyroid cancer has been studied to some extent (8-10); however, there are still a number of miRNAs that may be biologically relevant for the development of thyroid cancer.

Human miR-486 is situated on the short arm of chromosome 8 p11 within the ankyrin 1 gene and is transcribed from an intron (11). Genomic deletion of miR-486-5p has been associated with various cancers, including colorectal, lung, pancreatic and gastric cancer (12-16). However, to the best of our knowledge, only one study to date has investigated the function of miR-486-5p in thyroid cancer. Ma et al (17) demonstrated that the fibrillin 1 (FBN1) gene is a distinct molecular target 
for miR-486-5p in PTC cells. In addition, reduced miR-486-5p expression levels were identified in PTC tissues and cell lines, and miR-486-5p was demonstrated to suppress cell growth and enhance apoptosis in PTC. A limitation of this study was the small sample size; therefore, it is necessary to further explore the expression of miR-486-5p in thyroid cancer in a larger cohort to better understand the underlying molecular mechanisms.

The Cancer Genome Atlas (TCGA; https://cancergenome. nih.gov/) is a comprehensive database of key genetic mutations responsible for different types of cancer, which is generated by the National Cancer Institute and the National Human Genome Research Institute (18). The Gene Expression Omnibus (GEO; https://www.ncbi.nlm.nih.gov/geo/) was released by the National Center for Biotechnology Information in 2000 and is a public functional genomics data repository, which contains gene chip expression data as well as data from non-chip technologies, including serial analysis of gene and mass spectrometry (19). ArrayExpress (https://www.ebi.ac.uk/arrayexpress/) is an archive of functional genomics data from microarray and sequencing platforms (20). These databases offer an excellent source of information for studying the importance of miRNAs in PTC.

TCGA database was used to evaluate the expression levels of miR-486-5p in 507 patients with PTC and to analyze its association with clinical parameters. In addition, miR-486-5p expression data were extracted, and verified by GEO and ArrayExpress. Subsequently, the miR-486-5p target mRNAs were identified through 12 miRNA-mRNA prediction platforms. Finally, the potential molecular mechanisms by which miR-486-5p may contribute to PTC were investigated using bioinformatics tools.

\section{Materials and methods}

TCGA miR-486-5p data analysis. TCGA contains miRNA sequencing data from 507 PTC and 59 normal thyroid samples (21). In the present study, miR-486-5p expression data, as well as clinicopathological features from PTC vs. normal thyroid samples, were extracted from TCGA by the end of October 1, 2017. Follow-up cases over a 5-year period from October 1, 2012 were also included. The expression data for miR-486-5p were log 2 transformed and values $<1$ were censored. Student's t-test was conducted to compare the expression levels of miR-486-5p in 507 PTC and 59 normal thyroid samples, as well as the association between miR-486-5p expression and the clinical parameters from TCGA. The cut-off value for high and low miR-486-5p mRNA expression was based on median expression in the PTC group. Receiver operating characteristic (ROC) and summary receiver operating characteristic (SROC) curves were generated to evaluate the accuracy of miR-486-5p in identifying cancer and normal tissue. Kaplan-Meier (K-M) analysis and log-rank test were used to assess the prognostic value of miR-486-5p. SPSS version 22.0 (IBM Corp., Armonk, NY, USA) was utilized for statistical analyses and $\mathrm{P}<0.05$ was considered to indicate a statistically significant difference.

Validation of miR-486-5p expression using GEO and ArrayExpress. The GEO and ArrayExpress databases were searched for PTC-relevant miRNA chip or sequencing data using the following phrases: [(thyroid OR papillary OR follicular OR medullary) AND (tumor OR tumour OR cancer
OR carcinoma OR neopla* OR malignan*) AND (miRNA OR miR OR microRNA) AND (profil* OR array OR chip OR microarray OR microchip)]. "* refers to wildcard. All expression and clinical data related to miR-486-5p were extracted. Meta-analysis of GEO (GSE40807 (22), GSE57780 (unpublished data), GSE62054 (unpublished data), GSE73182 (23), ArrayExpress (E-MTAB-736) (24) and TCGA miRNA data was carried out in Stata version 12.0 (StataCorp LP, College Station, TX, USA). The pooled standard mean difference (SMD) with $95 \%$ confidence interval (CI) was utilized to assess miR-486-5p expression in PTC vs. normal thyroid tissue. $\chi^{2}$ and $\mathrm{I}^{2}$ statistics were calculated to measure the heterogeneity within the meta-analysis. The Mante-Haenszel fixed-effects model was applied if there was no obvious heterogeneity among the pooled studies ( $\chi^{2}$ test $\mathrm{P}>0.1$ and $\left.\mathrm{I}^{2}<50 \%\right)$; conversely, a random-effects model was applied when obvious heterogeneity was identified $\left(\chi^{2}\right.$ test $\mathrm{P}<0.1$ and $\mathrm{I}^{2}>50 \%$ ) (25).

Predicting miR-486-5p target genes. To identify potential miR-486-5p target genes, 12 miR-target prediction programs, including TDIANA-microTv4.0 (26), DIANA-microT-CDS (27), miRanda-rel2010 (28), miRBridge (29), miRDB4.0 (30), miRmap (31), miRNAMap (32), doRiNA (33), PITA (34), RNA22v2 (35), RNAhybrid2.1 (36) and Targetscan6.2 (37) were used. The $80 \mathrm{mRNAs}$ predicted by at least nine programs were considered potential miR-486-5p target mRNAs in PTC and used for further mRNA functional analysis.

Predicting miR-486-5p biological function. To evaluate the potential molecular mechanisms that are regulated by miR-486-5p-associated genes, FunRich version 3.0 software (http://www.funrich.org/) was used for Gene Ontology (GO) functional enrichment analysis (38). The software supports enrichment analysis of GO terms, including those involved in biological process, cellular component and molecular function, which can also be visualized. The results were considered statistically significant when the false discovery rate (FDR) was $<0.05$. The Database for Annotation, Visualization and Integrated Discovery (DAVID) version 6.8 (https://david. ncifcrf.gov/) and STRING version 10.5 (https://string-db.org/) were used for Kyoto Encyclopedia of Genes and Genomes (KEGG) enrichment analysis $(39,40)$. Genes in the top KEGG pathway were considered to be miR-486-5p hub genes.

Validation of the hub gene expression levels and their relationship with $\mathrm{miR}-486-5 \mathrm{p}$. The cBioPortal for Cancer Genomics (http://www.cbioportal.org/), which is a public resource for large-scale cancer genomics data, was used to explore hub gene expression in PTC tissues (41). To ascertain the correlation between miR-486-5p and hub genes, a Pearson's correlation analysis was conducted; $\mathrm{P}<0.05$ was considered to indicate a statistically significant difference. The expression of the hub genes in PTC and normal thyroid tissue was examined by immunohistochemistry via The Human Protein Atlas (http://www.proteinatlas.org/) (42).

\section{Results}

Downregulated miR-486-5p expression in PTC and its clinical significance. Data on miR-486-5p gene expression 
Table I. Association between miR-486-5p expression and clinicopathological factors in PTC.

\begin{tabular}{|c|c|c|c|c|}
\hline \multirow[b]{2}{*}{ Variable } & \multirow[b]{2}{*}{ No. } & \multicolumn{2}{|c|}{ Relative miR-486-5p expression } & \multirow[b]{2}{*}{ P-value } \\
\hline & & Mean \pm standard deviation & t-value & \\
\hline \multicolumn{5}{|l|}{ Tissue } \\
\hline Normal & 59 & $11.08 \pm 1.18$ & 13.81 & $<0.001$ \\
\hline PTC & 507 & $8.47 \pm 1.30$ & & \\
\hline \multicolumn{5}{|c|}{ Age (years) } \\
\hline$\leq 60$ & 393 & $8.61 \pm 1.27$ & 0.33 & 0.740 \\
\hline$>60$ & 113 & $8.56 \pm 1.36$ & & \\
\hline \multicolumn{5}{|l|}{ Sex } \\
\hline Female & 370 & $8.64 \pm 1.31$ & 1.28 & 0.203 \\
\hline Male & 136 & $8.48 \pm 1.24$ & & \\
\hline \multicolumn{5}{|l|}{ Stage } \\
\hline I-II & 336 & $8.72 \pm 1.30$ & 2.94 & 0.003 \\
\hline III-IV & 168 & $8.36 \pm 1.26$ & & \\
\hline \multicolumn{5}{|l|}{ Grade } \\
\hline I-II & 311 & $8.69 \pm 1.29$ & 1.92 & 0.056 \\
\hline III-V & 195 & $8.46 \pm 1.29$ & & \\
\hline \multicolumn{5}{|c|}{ Pathological lymph node } \\
\hline No & 231 & $8.72 \pm 1.28$ & 1.99 & 0.047 \\
\hline Yes & 225 & $8.49 \pm 1.20$ & & \\
\hline \multicolumn{5}{|c|}{ Metastasis } \\
\hline No & 496 & $8.61 \pm 1.29$ & 2.04 & 0.042 \\
\hline Yes & 9 & $7.73 \pm 0.99$ & & \\
\hline \multicolumn{5}{|l|}{ Tumor } \\
\hline No & 450 & $8.63 \pm 1.29$ & 2.51 & 0.012 \\
\hline Yes & 44 & $8.12 \pm 1.17$ & & \\
\hline \multicolumn{5}{|c|}{ Recurrence } \\
\hline No & 462 & $8.64 \pm 1.32$ & 2.41 & 0.016 \\
\hline Yes & 44 & $8.15 \pm 0.90$ & & \\
\hline
\end{tabular}

miR, microRNA; PTC, papillary thyroid carcinoma; SD, standard deviation.

from 507 PTC and 59 normal thyroid samples were extracted from TCGA. The expression levels of miR-486-5p were significantly decreased in PTC compared with in normal tissue $[8.47 \pm 1.30$ vs. $11.08 \pm 1.18 ; \mathrm{P}<0.001$; Fold change $(\mathrm{FC})=0.76$; Table I; Fig. 1A]. ROC curve analysis of miR-486-5p in discriminating PTC from normal tissue was calculated with an area under curve (AUC) of 0.918 ( $\mathrm{P}<0.001$; 95\% CI: 0.892-0.939; Fig. 1B). The miR-486-5p expression levels and clinicopathological factors are summarized in Table I. miR-486-5p expression levels were decreased in stage III-IV cancers $(8.36 \pm 1.26)$ compared with in stage I-II cancers $(8.72 \pm 1.30 ; \mathrm{P}=0.003)$. In addition, PTC tissues with pathological lymph nodes had decreased expression levels of miR-486-5p $(8.49 \pm 1.20)$ compared with PTC tissues without pathological lymph nodes $(8.72 \pm 1.28 ; \mathrm{P}=0.047)$. Furthermore, miR-486-5p was downregulated in tissues with metastasis $(7.73 \pm 0.99)$ compared with in tissues without metastasis $(8.61 \pm 1.29 ; \mathrm{P}=0.042)$. Decreased expression levels of miR-486-5p were observed in PTC group (8.12 \pm 1.17$)$ compared with the normal thyroid group (8.63 \pm 1.29 ; $\mathrm{P}=0.012)$. miR-486-5p expression was also reduced in recurrent cancer $(8.15 \pm 0.90)$ compared with non-recurrent cancer (8.64 \pm 1.32 ; $\mathrm{P}=0.016)$. Differential miR-486-5p expression was not observed for the remaining clinicopathological factors analyzed (Table I). Subsequently, the prognostic value of miR-486-5p expression was investigated. The K-M survival curves indicated that the median overall survival (OS) for the high expression group was 1,443 days, whereas the median OS for the low expression group was 1,015 days. The curves suggested that PTC cases with higher miR-486-5p expression levels were likely to have an improved clinical outcome. However, there was no statistically significant difference in the OS [log-rank $\mathrm{P}=0.355$; hazard ratio $(\mathrm{HR})=0.617$; $95 \% \mathrm{CI}$ : 0.221-1.718; Fig. 2A] and the disease-free survival (DFS) (log-rank P=0.132; HR=0.454; 95\% CI: 0.162-1.269; Fig. 2B) of samples with high vs. low expression levels of miR-486-5p (cut-off $=8.469$ ). Therefore, the ability for miR-486-5p expression to predict OS and DFS is limited. 
A

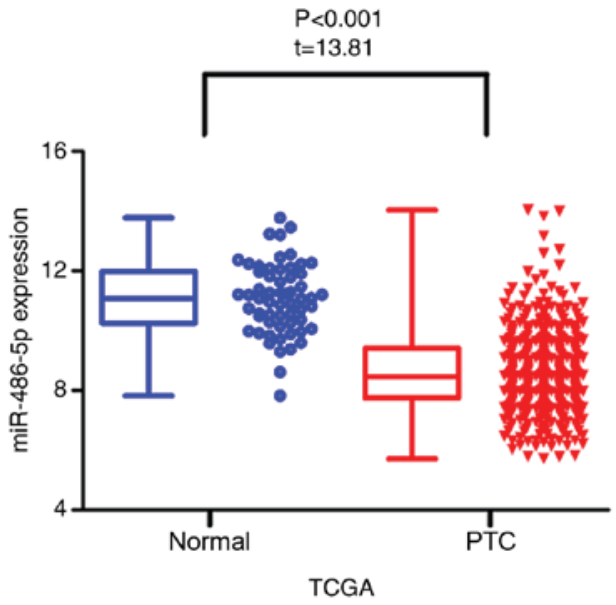

B

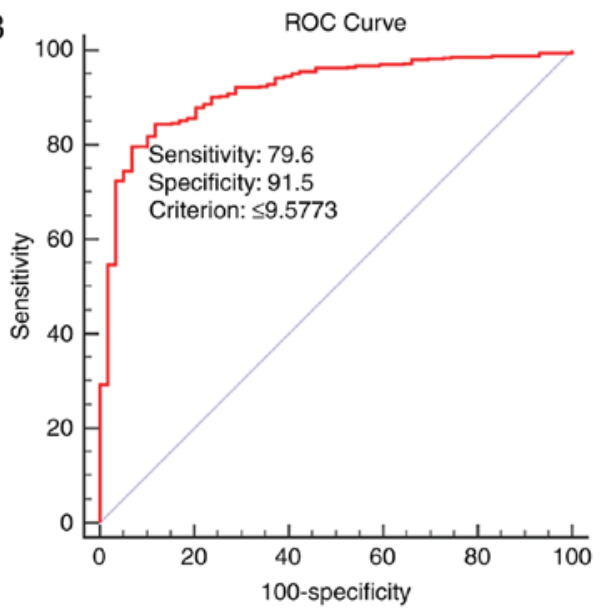

Figure 1. (A) Relative expression levels of miR-486-5p in PTC vs. normal thyroid tissues using the dataset from TCGA. (B) ROC curve analysis of miR-486-5p in discriminating PTC from normal controls. miR, microRNA; PTC, papillary thyroid carcinoma; ROC, receiver-operating characteristic; TCGA, The Cancer Genome Atlas.
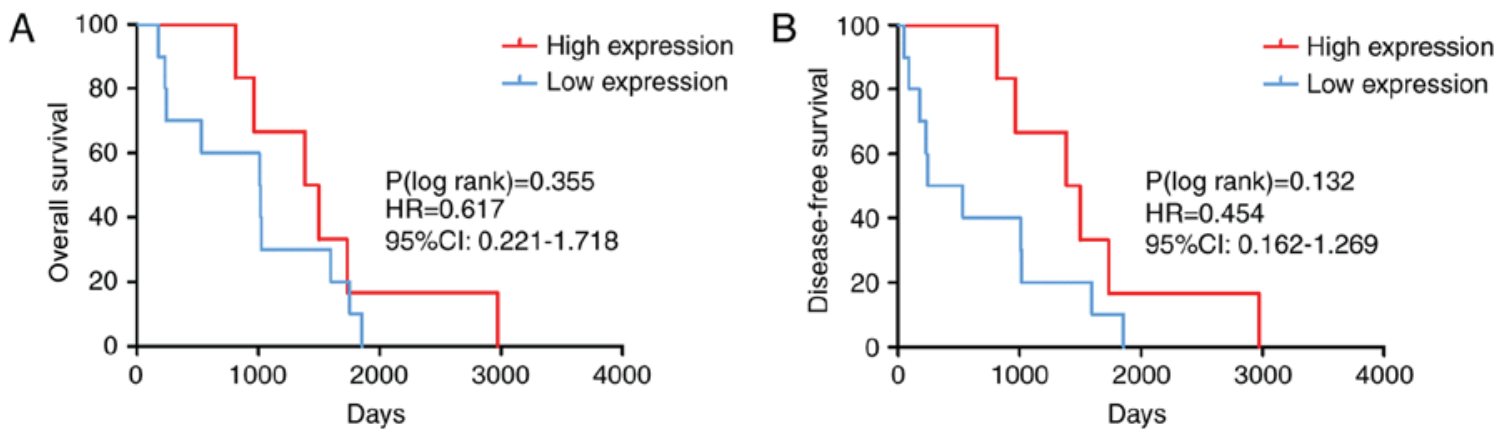

Figure 2. Survival curves in patients with PTC according to miR-486-5p expression levels (cut-off=8.469). Kaplan-Meier survival analysis of (A) overall survival and (B) disease-free survival in 507 PTC samples. CI, confidence interval; HR, hazard ratio; miR, microRNA; PTC, papillary thyroid carcinoma.

Verification of miR-486-5p downregulation in PTC using GEO and ArrayExpress data. GSE40807, GSE57780, GSE62054 and GSE73182 were obtained from the GEO database, which provided miR-486-5p expression levels in 77 PTC and 56 normal thyroid tissues (Fig. 3). The expression levels of miR-486-5p were lower in PTC compared with in normal samples in the

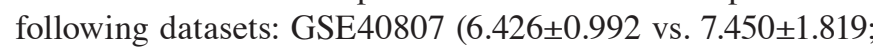
$\mathrm{P}=0.003$; $\mathrm{FC}=1.159$; Fig. 3A), GSE62054 (5.000 \pm 1.003 vs. $6.176 \pm 1.349 ; \mathrm{P}=0.022 ; \mathrm{FC}=0.810 ;$ Fig. $3 \mathrm{E})$ and $\mathrm{GSE73182}$ (9.716 \pm 0.171 vs. $9.952 \pm 0.173 ; \mathrm{P}=0.012 ; \mathrm{FC}=0.976$; Fig. $3 \mathrm{G})$. The expression levels of miR-486-5p did not differ significantly between PTC and normal tissue in the GSE57780 dataset $(10.648 \pm 0.133$ vs. $12.195 \pm 0.994 ; \mathrm{P}=0.056 ; \mathrm{FC}=0.873$; Fig. 3C). Chip array data from a single study (E-MTAB-736) was obtained from ArrayExpress and the data also indicated that miR-486-5p expression levels were downregulated in the 12 PTC samples compared with in the 10 normal tissue samples $(8.048 \pm 0.593$ vs. $8.914 \pm 0.579 ; \mathrm{P}=0.003 ; \mathrm{FC}=0.903$; Fig. 4A). To evaluate the ability of miR-486-5p to distinguish cancer from normal thyroid samples, ROC curve analyses were performed. The AUC values for miR-486-5p calculated using the GSE40807, GSE57780, GSE62054, GSE73182 and E-MTAB-736 datasets were 0.684 ( $\mathrm{P}<0.001$; cut-off $\leq 7.217$; Fig. 3B), 1.000 ( $\mathrm{P}<0.001$; cut-off $\leq 10.747$; Fig. 3D), 0.746 $(\mathrm{P}=0.036$; cut-off $\leq 5.644$; Fig. $3 \mathrm{~F}), 0.884$ ( $\mathrm{P}<0.001$; cut-off

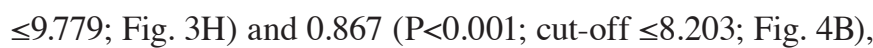
respectively.

Further verification of miR-486-5p downregulation in PTC using SMD and SROC. miR-486-5p expression data from TCGA, GEO (GSE40807, GSE57780, GSE62054 and GSE73182) and ArrayExpress (E-MTAB-736) were combined for meta-analysis, which included a total of 603 PTC and 125 normal tissue samples (Table II). The pooled SMD of miR-486-5p was -1.358 [95\% CI, -1.950-(-0.766); $\mathrm{P}<0.001$; Fig. 5A] by the random effects model, and the $\mathrm{P}$-value of the heterogeneity test was $0.001\left(\mathrm{I}^{2}=75.6 \%\right)$. From meta-analysis, the AUC of the SROC was 0.84 (95\% CI, 0.81-0.87; $\mathrm{P}<0.001$; Fig. 5B).

Prediction of miR-486-5p target mRNAs and GO analysis. From at least nine of the 12 miRNA target prediction databases, $80 \mathrm{mRNA}$ targets of miR-486-5p were identified. To understand the biological function of miR-486-5p in PTC, GO enrichment analysis of genes encoding for miR-486-5p target mRNAs was performed in FunRich. 'Regulation of nucleobase, nucleoside, nucleotide and nucleic acid metabolism' ( $\mathrm{P}=0.008$; Fig. 6A), 'Cytoplasm' ( $\mathrm{P}=6.158 \times 10^{-8}$; Fig. 6B) and 'RNA binding' $(\mathrm{P}=0.004$; Fig. 6C) were the top functions for biological process, cellular component and molecular function, respectively. 


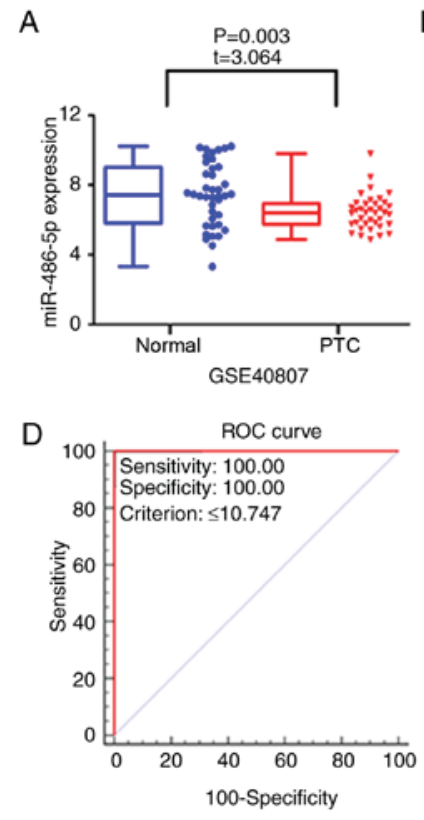

G

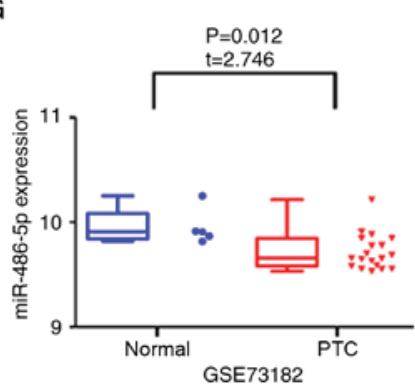

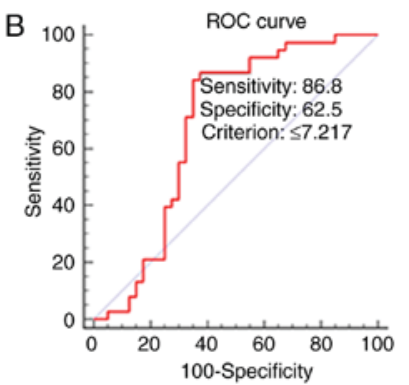
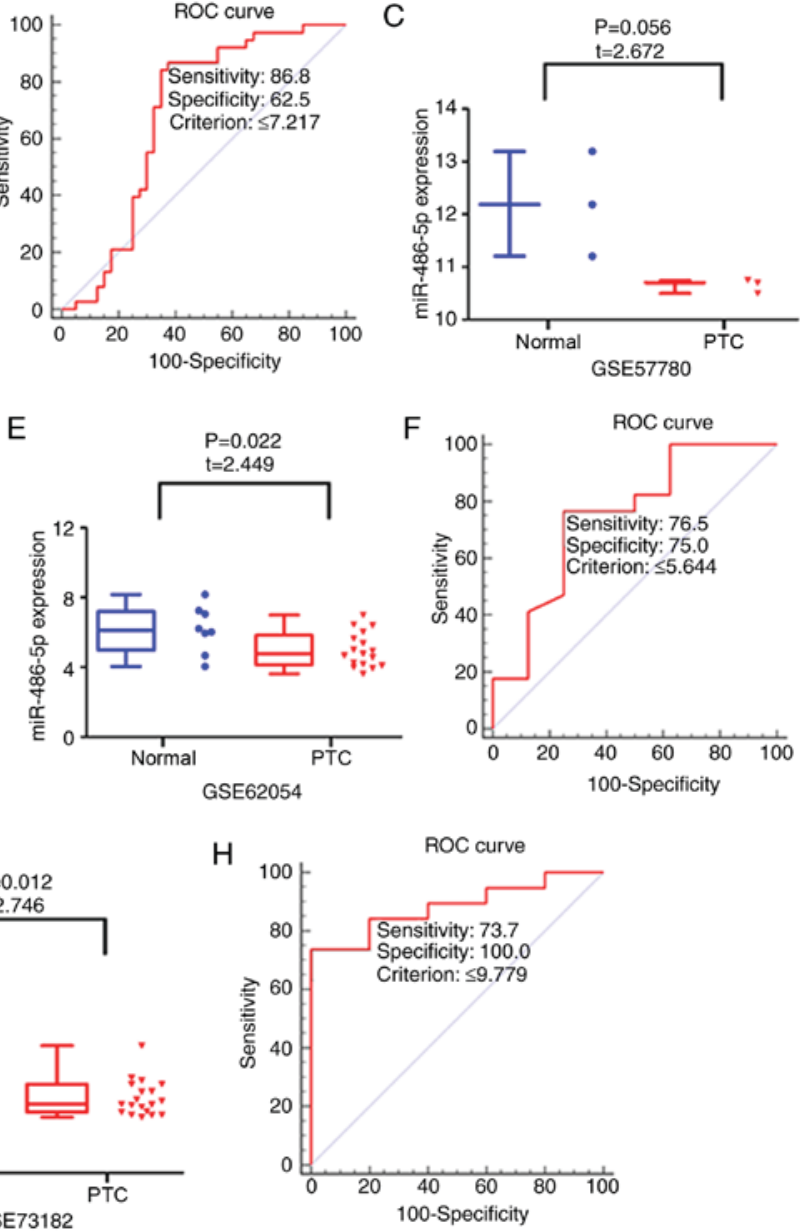

Figure 3. Relative miR-486-5p expression levels in PTC and normal tissue using the datasets (A) GSE40807, (C) GSE57780, (E) GSE62054 and (G) GSE73182. ROC curve analysis of miR-486-5p in discriminating PTC from normal controls using the datasets (B) GSE40807, (D) GSE57780, (F) GSE62054 and (H) GSE73182. miR, microRNA; PTC, papillary thyroid carcinoma; ROC, receiver-operating characteristic.

Identification of miR-486-5p-regulated pathways. The KEGG pathway enrichment analysis was performed on miR-486-5p-relevant genes using DAVID. The results revealed the most significantly enriched pathway was 'hsa05200: Pathways in cancer' with six genes including CRK like proto-oncogene (CRKL), C-terminal binding protein 2, forkhead box O1, phosphatase and tensin homolog (PTEN), phosphoinositide-3-kinase regulatory subunit 1 and tropomyosin 3 (TPM3) $(\mathrm{P}=0.010$; Fig. 7). In addition, protein-protein interaction network analysis was performed on the 80 relevant genes using STRING: 'hsa05206: MicroRNAs in cancer' was identified, with six genes including CRKL, pim-1 proto-oncogene, sirtuin 1, PTEN, SRY-box 4 and TPM3 (Fig. 8). The three common genes (CRKL, PTEN and TPM3) of the 'hsa05200: Pathways in cancer' and 'hsa05206: MicroRNAs in cancer' pathways from the analyses were considered hub genes (Fig. 9A).

Validation of hub genes. To validate the relationship between miR-486-5p and the hub genes CRKL, PTEN and TPM3, their gene expression levels were examined using the cBioPortal for Cancer Genomics. The expression levels of the three hub genes (CRKL, PTEN and TPM3) in PTC were markedly higher than in non-cancerous thyroid samples (Fig. 9B). Pearson's correlation analysis was performed to assess the relationship between miR-486-5p and the hub genes. A statistically significant negative correlation between miR-486-5p and CRKL ( $\mathrm{r}=-0.111$; $\mathrm{P}=0.013$; Fig. 9C) and TPM3 (r=-0.089; $\mathrm{P}=0.044$; Fig. 9D) was observed. Finally, the protein expression levels of CRKL, PTEN and TPM3 in PTC tissues were visualized using immunohistochemical staining images from The Human Protein Atlas database. In line with the gene expression data, protein expression of CRKL, PTEN and TPM3 was increased in PTC compared with in normal thyroid tissue (Fig. 10).

\section{Discussion}

In the present study, significantly decreased expression levels of miR-486-5p in PTC were demonstrated by analysis of TCGA, GEO (GSE40807, GSE57780, GSE62054 and GSE73182) and ArrayExpress (E-MTAB-736) data, which included 603 PTC and 125 normal thyroid tissue samples. Downregulation of miR-486-5p was associated with cancer stage, pathological lymph node, metastasis, neoplasm and disease recurrence, all of which are clinical indicators of poor disease outcome. SROC curve analysis of miR-486-5p data from TCGA, GEO (GSE40807, GSE57780, GSE62054 and GSE73182) and ArrayExpress (E-MTAB-736) demonstrated that expression levels could provide high diagnostic value for PTC with an AUC of 0.84 (95\% CI: 0.81-0.87). In addition, downregulation of miR-486-5p in patients with PTC may lead to worse 
OS. The biological functions of miR-486-5p in facilitating the onset and progression of PTC were also explored. The potential miR-486-5p target mRNAs were found to be enriched in diverse signaling pathways, including 'hsa05200: Pathways in cancer' and 'hsa05206: MicroRNAs in cancer'. Furthermore, three hub genes, CRKL, PTEN and TPM3, were identified, all of which were notably upregulated in PTC samples based on analysis using the cBioPortal for Cancer Genomics. Therefore, it may be hypothesized that downregulated miR-486-5p serves as a clinical biomarker for PTC and exerts a potential biological role in progression of the disease.

There is accumulating evidence suggesting that aberrant miRNA expression levels contribute towards PTC development and progression. Several studies have identified a crucial role for miR-486-5p during onset and progression of various types of cancer; for example, miR-486-5p is markedly downregulated in non-small cell lung cancer, osteosarcoma, breast cancer, myxoid liposarcoma and pancreatic ductal adenocarcinomas (43-47). Conversely, miR-486-5p is upregulated in chronic myelocytic leukemia and myeloid leukemia of Down syndrome $(48,49)$. Therefore, miR-486-5p may be differentially expressed depending on cancer type. To the best of our knowledge, only one study has explored miR-486-5p expression levels in PTC. Ma et al (50) demonstrated that miR-486-5p was predominantly downregulated in PTC tissues and cell lines, whereas miR-486-5p upregulation resulted in suppression of proliferation and growth by targeting FBN1. However, a limitation to this study is that it only analyzed 20 paired PTC and normal thyroid samples by reverse transcription-quantitative polymerase chain reaction (50). In the present study, integration of results from multiple studies via bioinformatics analysis revealed that miR-486-5p expression levels were decreased in PTC, and may have diagnostic and prognostic value for patients with PTC. Further investigations in vivo and in vitro are required to identify the underlying molecular mechanism of miR-486-5p in PTC. Exploring miR-486-5p expression levels in neoplastic tissues from patients with PTC will aid in understanding its clinical value.

miR-486-5p is upregulated by miR-660-5p via the miR-660-5p-mouse double minute 2 homolog-proto-oncogene-p53 axis in lung cancer (51). In gastric cancer, miR-486-5p acts as a tumor suppressor and its downregulation facilitates tumor growth by increasing transcription of the anti-apoptotic factor, olfactomedin 4 (52). Similarly, miR-486-5p enhances hepatocellular carcinoma chemosensitivity to sorafenib and regulates metastasis by targeting citron rho-interacting serine/threonine kinase and claudin 10 (53). In vitro, Borjigin et al (46) revealed that the FUS RNA binding protein-DNA damage inducible transcript 3 fusion protein suppressed miR-486-5p expression leading to induction of plasminogen activator inhibitor-1 expression in human myxoid liposarcoma. Overall, these results suggest that miR-486-5p may act as a tumor suppressor in various types of cancer. However, most of the aforementioned studies only investigated individual mRNA targets of miR-486-5p, whereas the present study utilized pathway enrichment and network analysis to identify possible targets of miR-486-5p. Certain target genes were associated with 'Regulation of nucleobase, nucleoside, nucleotide and nucleic acid metabolism', 'Cytoplasm' and 'RNA binding' in the GO functional enrichment analysis. Notably, the three genes CRKL,

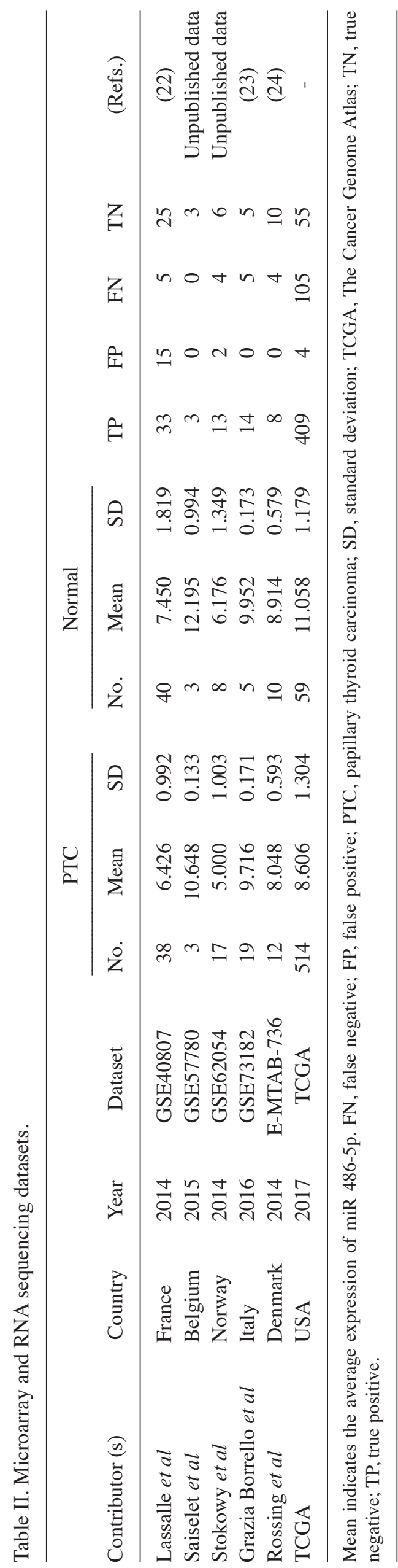


A

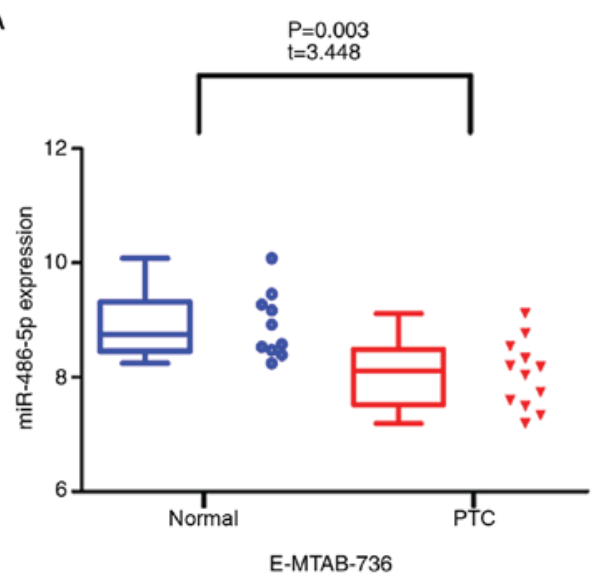

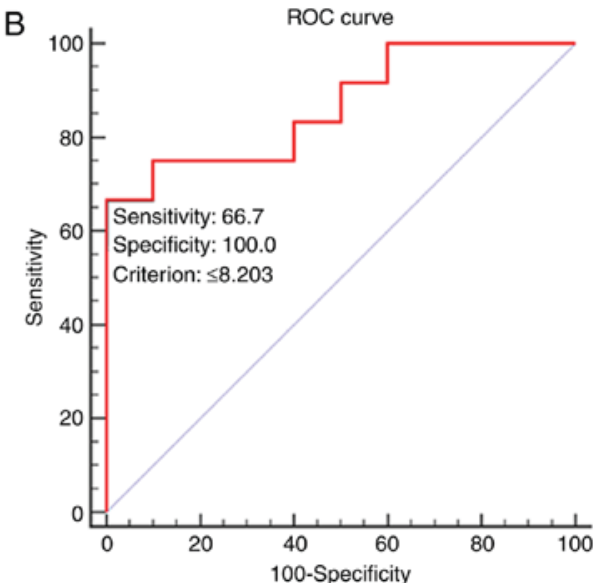

Figure 4. (A) Relative expression levels of miR-486-5p in PTC vs. normal thyroid tissue using the E-MTAB-736 dataset from ArrayExpress. (B) ROC curve analysis of miR-486-5p in discriminating PTC from normal controls. CI, confidence interval; miR, microRNA; PTC, papillary thyroid carcinoma; ROC, receiver-operating characteristic; SENS, sensitivity; SMD, standard mean difference; SPEC, specificity; TCGA, The Cancer Genome Atlas.

A

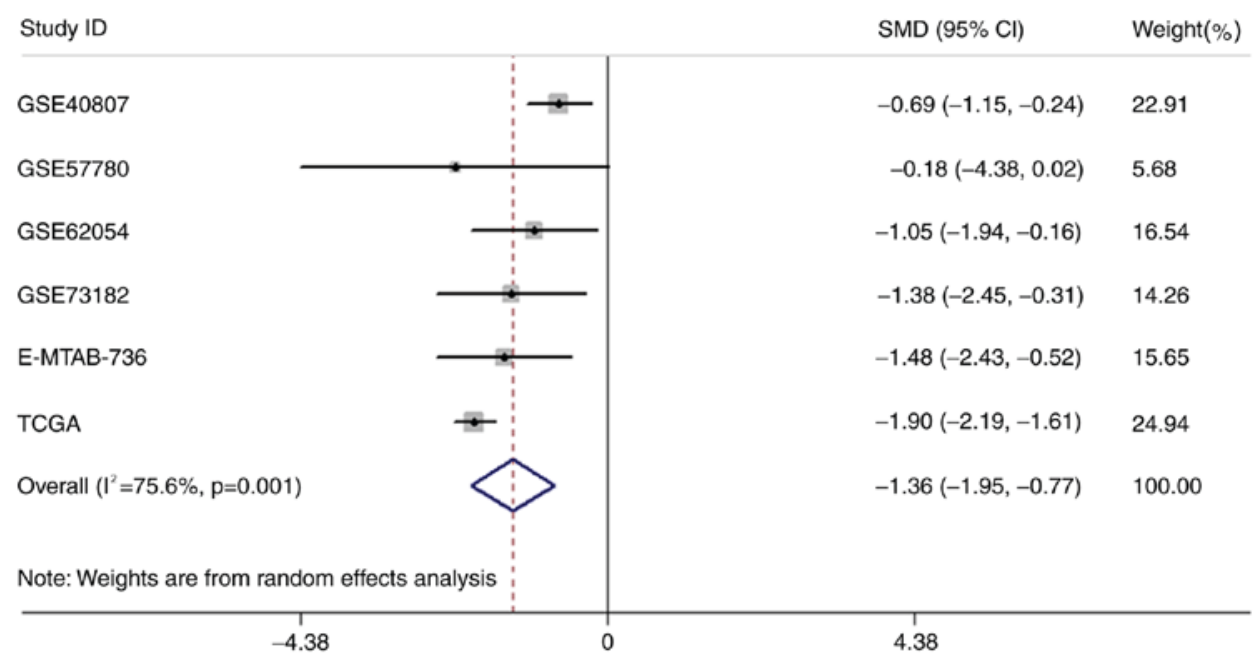

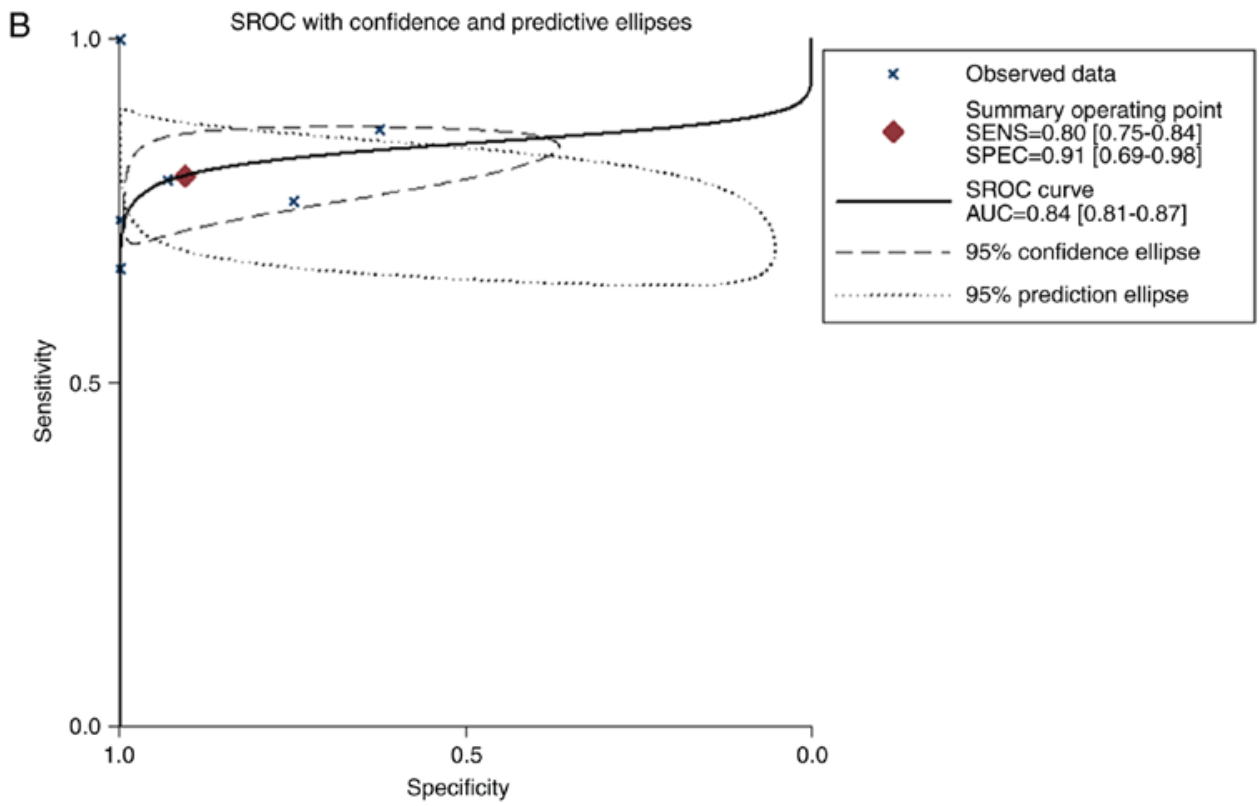

Figure 5. Meta-analysis of miR-486-5p expression levels in PTC. (A) Forest plot of all datasets evaluating miR-486-5p expression between PTC and normal thyroid tissue. (B) SROC curve for miR-486-5p in the diagnosis of PTC for all studies. Datasets from TCGA, GSE40807, GSE57780, GSE62054, GSE73182 and E-MTAB-736 were included in the meta-analysis. AUC, area under curve; CI, confidence interval; miR, microRNA; PTC, papillary thyroid carcinoma; SROC, summary receiver operating characteristic; TCGA, The Cancer Genome Atlas. 
A

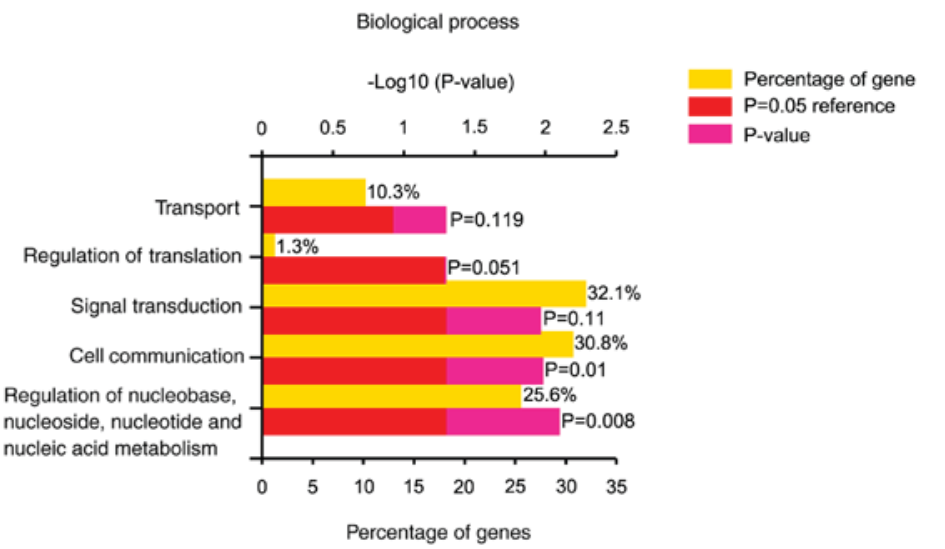

B
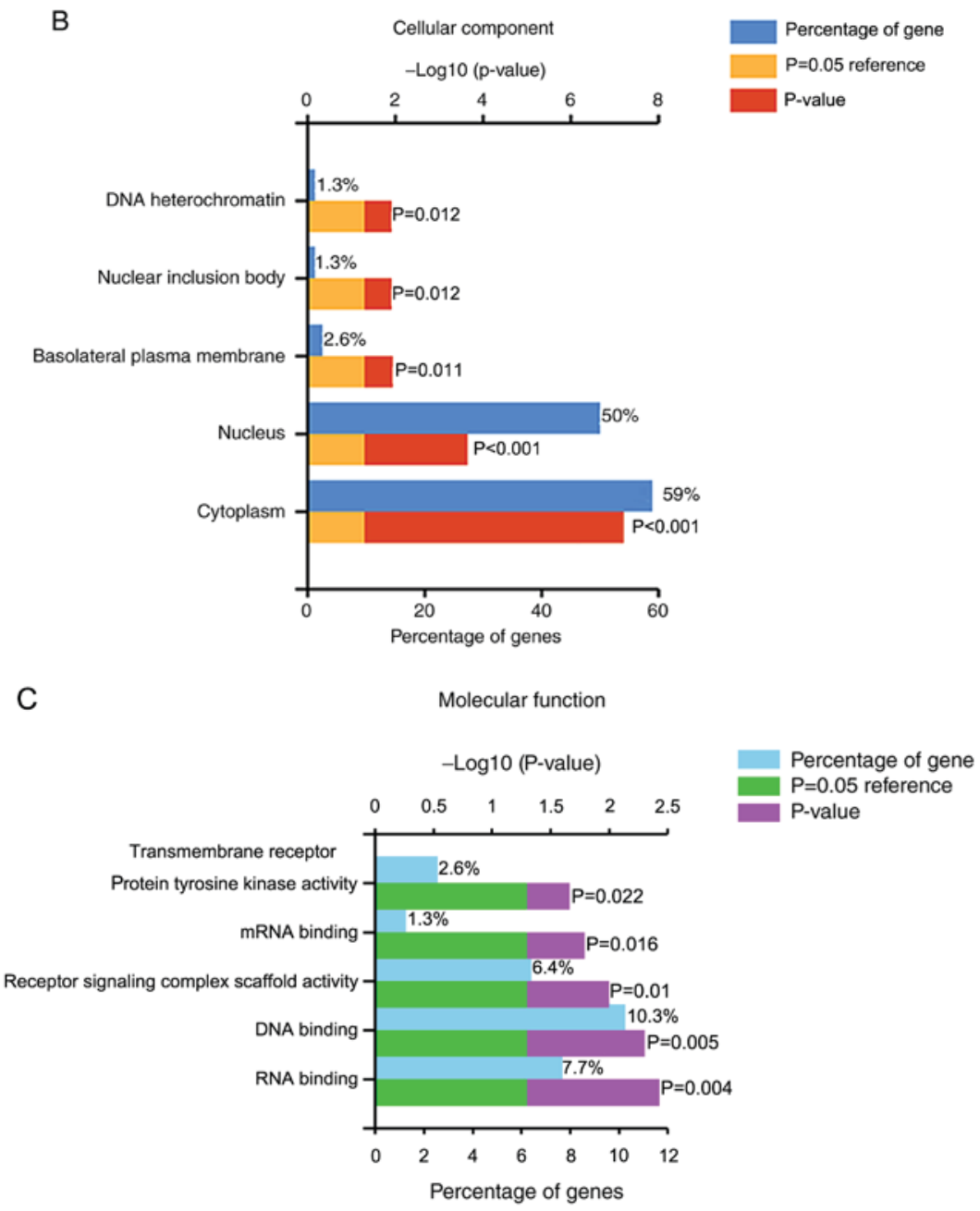

Figure 6. Functional enrichment analysis of potential microRNA-486-5p target genes in the Gene Ontology categories (A) biological process, (B) cellular component and (C) molecular function.

PTEN and TPM3 were identified in both 'hsa05200: Pathways in cancer' and 'hsa05206: MicroRNAs in cancer' through KEGG pathway enrichment analysis; therefore, these genes may be essential to the pathogenesis and progression of PTC.

CRKL encodes for a substrate of the BCR-ABL proto-oncogene fusion gene, and has a pivotal role in fibroblast transformation and may be oncogenic (54). Yang et al (55) reported that CRKL is associated with cervical lymph node metastasis and invasion of capsules in PTC, which has clinical implications on treatment. PTEN acts as an oncogene and is highly prevalent in various cancers (56-58). Fang et al (59) demonstrated that ribonucleotide reductase catalytic subunit M1 induces PTEN expression and decreases Akt serine/ threonine kinase phosphorylation in a ribonucleotide reductase activity-independent manner in PTC. In addition, Zhao et al (60) demonstrated that PTEN mRNA levels may serve as a biomarker for PTC onset and act as a molecular diagnostic index. TPM3 encodes a member of the tropomyosin family of actin-binding proteins and is frequently involved in genetic rearrangements resulting in fusion with the neurotrophic 


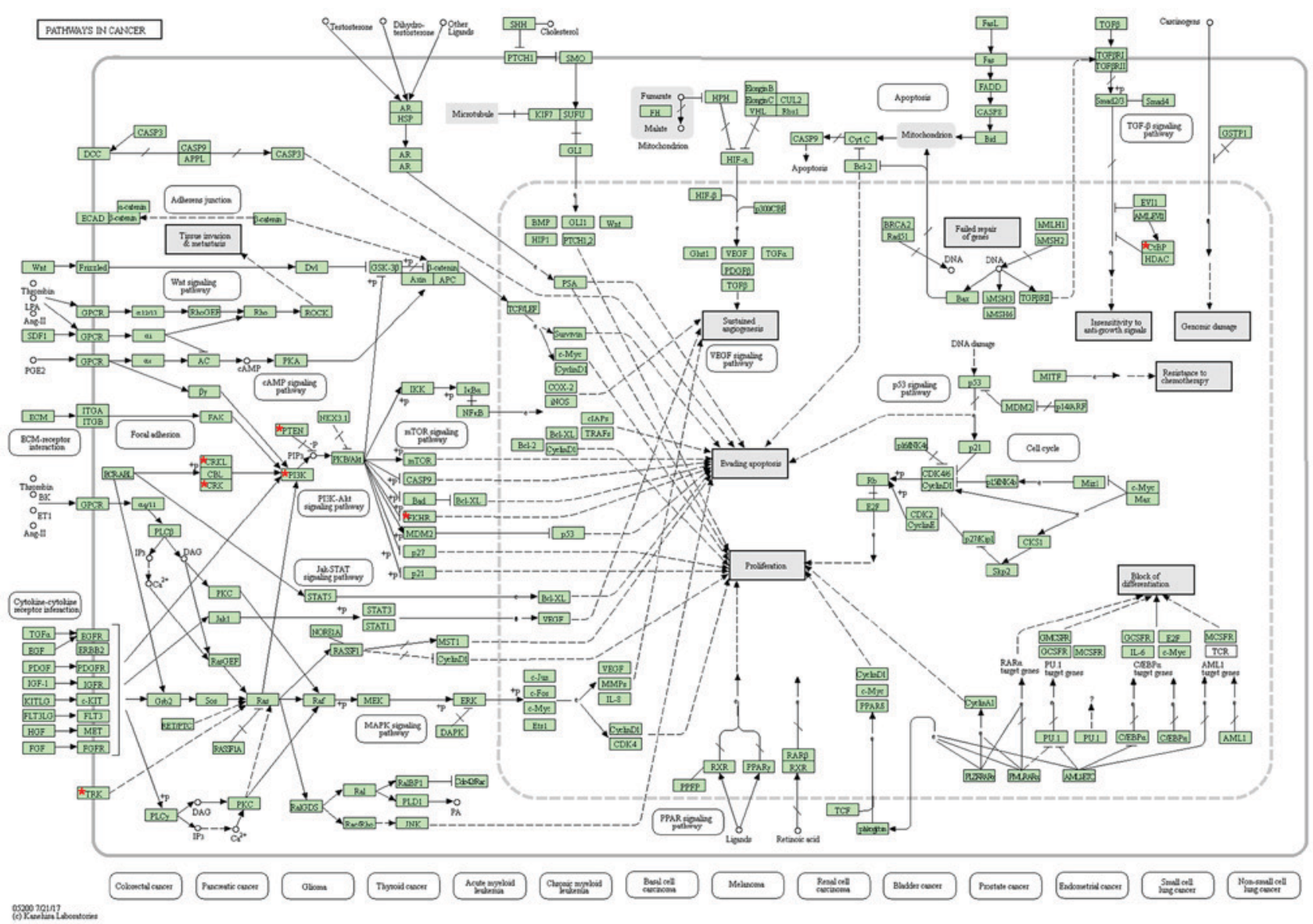

Figure 7. Kyoto Encyclopedia of Genes and Genomes pathway map illustrating 'hsa05200: Pathways in cancer', as identified by pathway enrichment analysis using the Database for Annotation, Visualization and Integrated Discovery. Enriched target genes of miR-486-5p are shown in red. miR, microRNA.

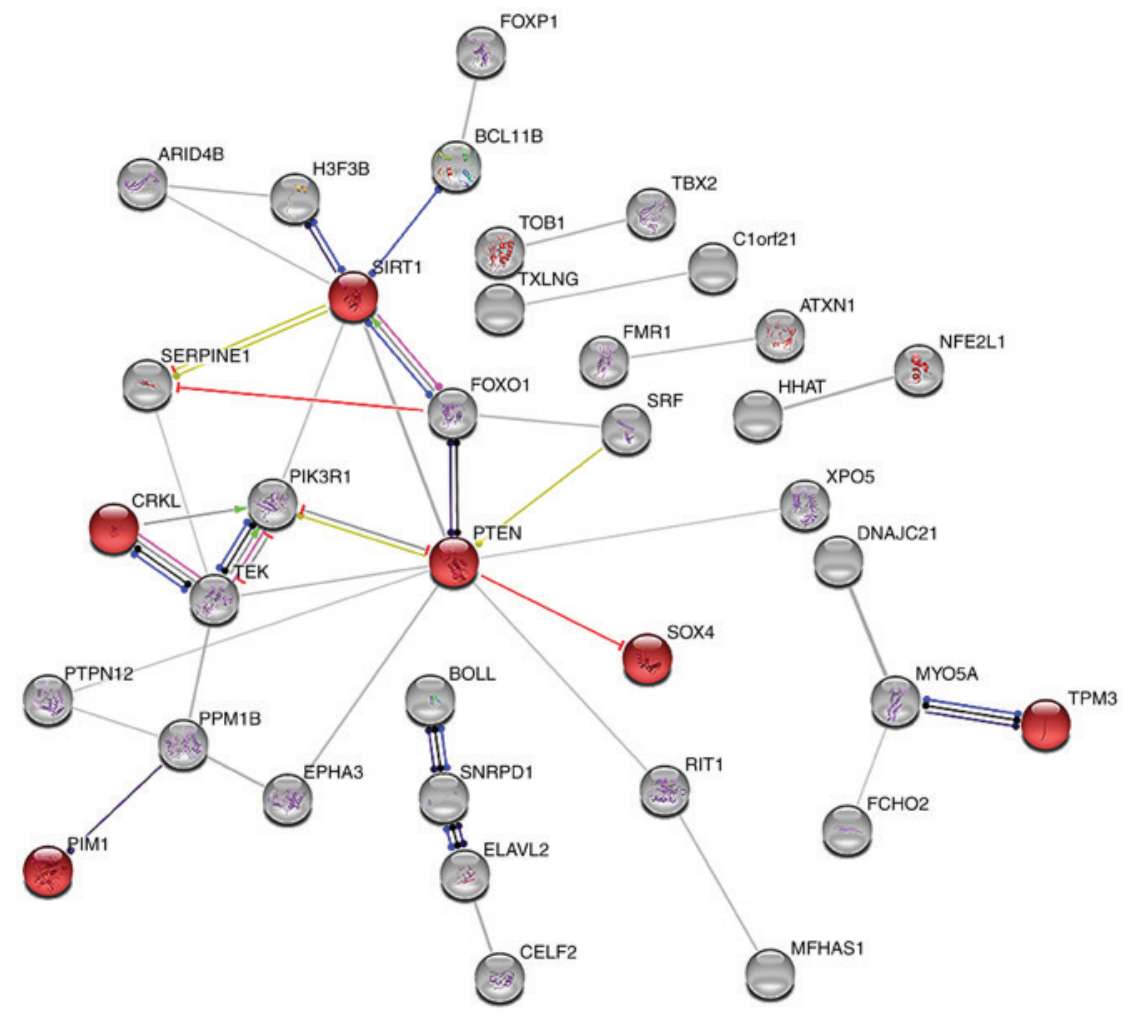

Figure 8. Kyoto Encyclopedia of Genes and Genomes pathway map illustrating 'hsa05206: MicroRNAs in cancer', as identified by protein-protein interaction network analysis using STRING. The network map was generated in the Database for Annotation, Visualization and Integrated Discovery 6.8. Enriched target genes of miR-486-5p are shown in red. miR, microRNA. 


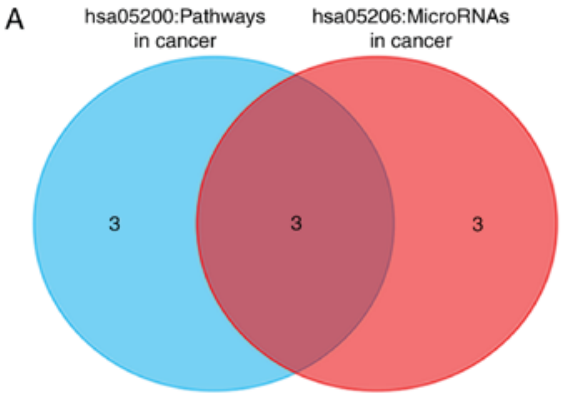

C

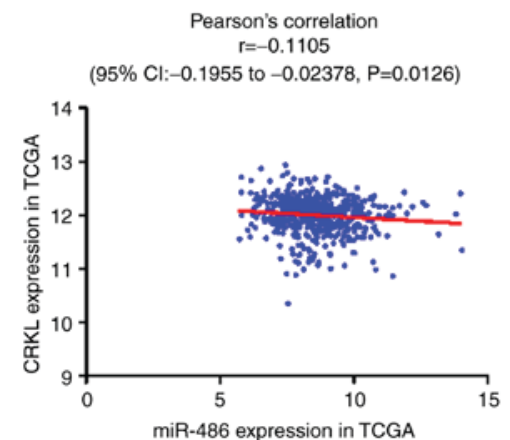

B

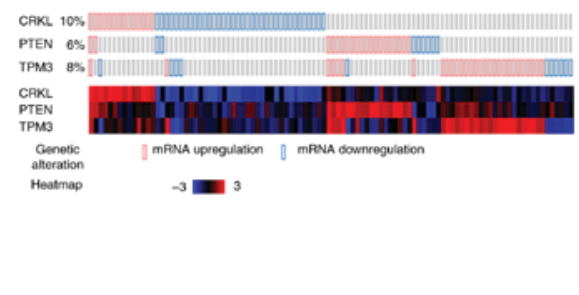

D

Pearson's correlation $r=-0.0894$

(95\% Cl:-0.1749 to-0.0024, $\mathrm{P}=0.0439)$

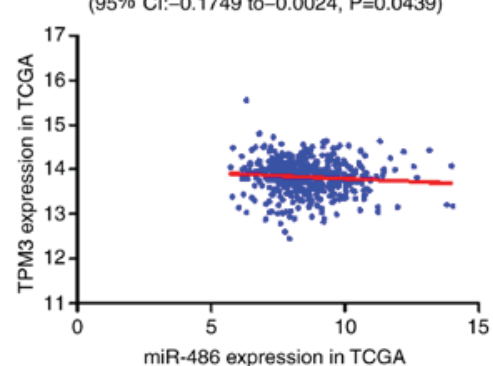

Figure 9. Expression of the hub genes CRKL, PTEN and TPM3 in PTC. (A) Venn diagram showing overlap between predicted miR-486-5p target genes in the 'hsa05200: Pathways in cancer' and 'hsa05206: MicroRNAs in cancer'. (B) Expression levels of CRKL, PTEN and TPM3 using the cBioPortal for cancer genomics using data from TCGA. The percentages indicate the proportion of HCC patients $(n=371)$ with genetic alterations detected. Pearson's correlation between (C) CRKL or (D) TPM3 and miR-486-5p expression using data from TCGA. CI, confidence interval; CRKL, CRK like proto-oncogene; miR, microRNA; PTC, papillary thyroid carcinoma; PTEN, phosphatase and tensin homolog; TCGA, The Cancer Genome Atlas; TPM3, tropomyosin 3.
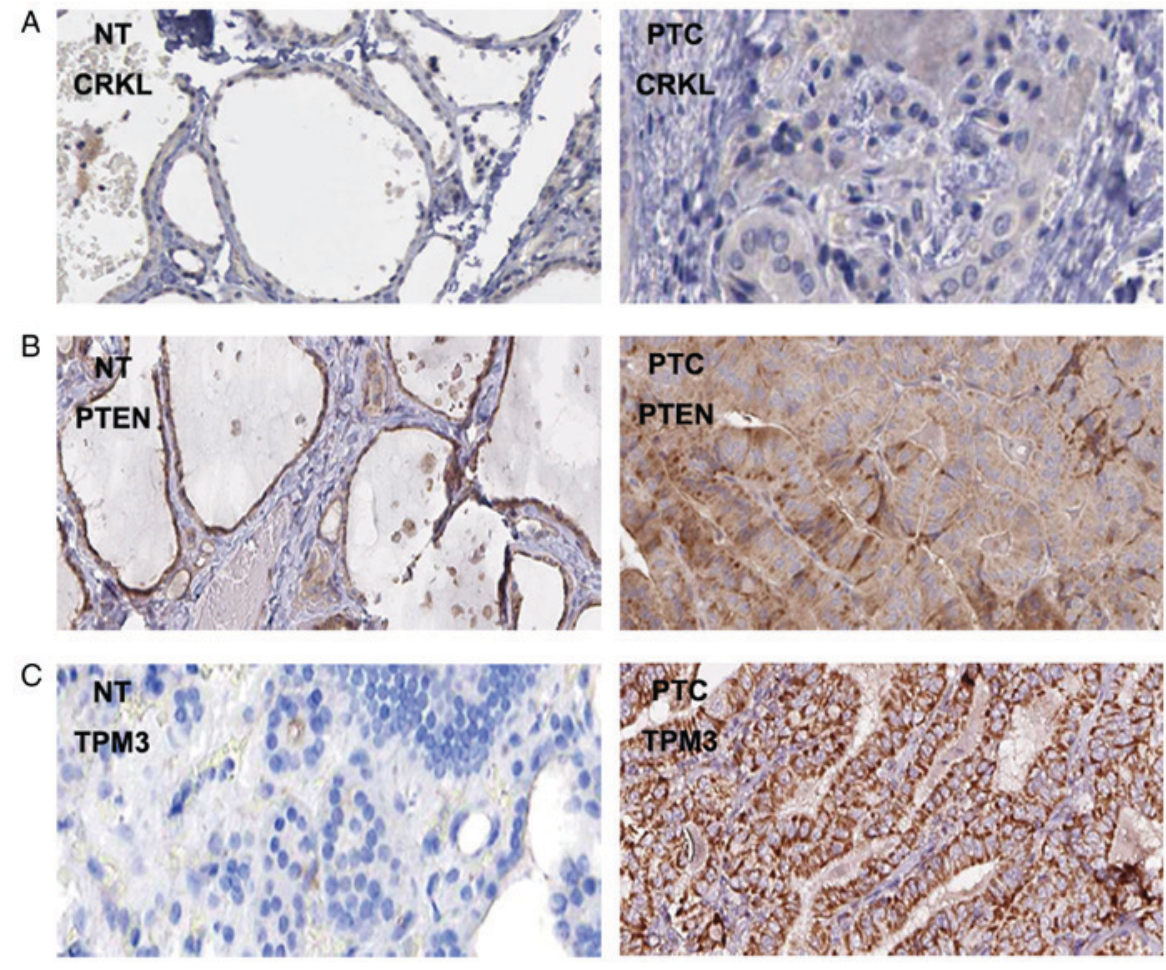

Figure 10. Immunohistochemical staining images from The Human Protein Atlas of (A) CRKL (https://www.proteinatlas.org/search/CRKL), (B) PTEN (https://www.proteinatlas.org/search/PTEN) and (C) TPM3 (https://www.proteinatlas.org/search/TPM3) in PTC vs. NT tissue. CRKL, CRK like proto-oncogene; NT, normal thyroid; PTC, papillary thyroid carcinoma; PTEN, phosphatase and tensin homolog; TPM3, tropomyosin 3.

tyrosine kinase receptor type 1 (NTRK1) gene, which then acts as an oncogene (61). Numerous studies that have demonstrated the association of NTRK1 with genetic rearrangements in PTC $(62,63)$.
Notably, CRKL, PTEN and TPM3 were more highly expressed in PTC, and the results suggested that these genes may be regulated by miR-486-5p, whose expression is lower in PTC. It may be hypothesized that CRKL, PTEN and TPM3 have an 
important role in PTC and are potential molecular targets in the clinic. Nevertheless, in silico target gene prediction algorithms have limited specificity, and further in vitro and in vivo investigations are required to confirm the function of miR-486-5p in PTC.

In conclusion, the present study revealed that the expression of miR-486-5p was significantly downregulated in PTC. The clinical implications of miR-486-5p were also evaluated using results from multiple datasets. Bioinformatics analysis was performed to elucidate the molecular mechanisms of miR-486-5p in facilitating the onset and progression of PTC. Three hub genes CRKL, PTEN and TPM3, were identified, which may be potential targets of miR-486-5p in PTC. The clinical importance of miR-486-5p in PTC and its underlying molecular mechanisms require further investigation; however, the results indicated that miR-486-5p may be involved in the pathogenesis of PTC.

\section{Acknowledgements}

The authors thank TCGA, GEO, ArrayExpress and The Human Protein Atlas for providing the data.

\section{Funding}

The present study was partly supported by the Guangxi Scientific Research and Technology Development Plan (grant no. 1598011-4), the Fund of Guangxi Key R\&D Project Plan (grant no. AB17195020) and the Fund of National Natural Science Foundation of China (grant nos. NSFC81060202 and NSFC81260222).

\section{Availability of data and materials}

The datasets used and/or analyzed during the current study are available from the corresponding author on reasonable request.

\section{Authors' contributions}

D-YW, HY, GC and YH designed the study and performed the experiments. D-HP, PL, Q-YM, Y-HL, J-QC, GC and Y-PW participated in data processing and statistical analysis. D-YW and Y-HL wrote the manuscript. D-YW, Y-HL, YH and HY critically revised the manuscript. All authors read and approved the final manuscript.

\section{Ethics approval and consent to participate}

Not applicable.

\section{Patient consent for publication}

Not applicable.

\section{Competing interests}

The authors declare that they have no competing interests.

\section{References}

1. Kohlhapp FJ, Mitra AK, Lengyel E and Peter ME: MicroRNAs as mediators and communicators between cancer cells and the tumor microenvironment. Oncogene 34: 5857-5868, 2015.
2. Vigneri R, Malandrino P and Vigneri P: The changing epidemiology of thyroid cancer: Why is incidence increasing? Curr Opin Oncol 27: 1-7, 2015.

3. Qiu Z, Li H, Wang J and Sun C: miR-146a and miR-146b in the diagnosis and prognosis of papillary thyroid carcinoma. Oncol Rep 38: 2735-2740, 2017.

4. Penha RCC, Sepe R, De Martino M, Esposito F, Pellecchia S, Raia M, Del Vecchio L, Decaussin-Petrucci M, De Vita G, Pinto LFR and Fusco A: Role of Dicer1 in thyroid cell proliferation and differentiation. Cell Cycle 16: 2282-2289, 2017.

5. Zhuang J, Ye Y, Wang G, Ni J, He S, Hu C, Xia W and Lv Z: MicroRNA-497 inhibits cellular proliferation, migration and invasion of papillary thyroid cancer by directly targeting AKT3. Mol Med Rep 16: 5815-5822, 2017.

6. Mahmoudian-Sani MR, Mehri-Ghahfarrokhi A, Asadi-Samani $M$ and Mobini GR: Serum miRNAs as biomarkers for the diagnosis and prognosis of thyroid cancer: A comprehensive review of the literature. Eur Thyroid J 6: 171-177, 2017.

7. Boufraqech M, Klubo-Gwiezdzinska $J$ and Kebebew E: MicroRNAs in the thyroid. Best Pract Res Clin Endocrinol Metab 30: 603-619, 2016.

8. Bai D, Sun H, Wang X, Lou H, Zhang J, Wang X and Jiang L: MiR-150 inhibits cell growth in vitro and in vivo by restraining the RAB11A/WNT/ $\beta$-catenin pathway in thyroid cancer. Med Sci Monit 23: 4885-4894, 2017.

9. Wang J, Yang H, Si Y, Hu D, Yu Y, Zhang Y, Gao M and Zhang H: Iodine promotes tumorigenesis of thyroid cancer by suppressing Mir-422a and Up-regulating MAPK1. Cell Physiol Biochem 43: 1325-1336, 2017.

10. Paiva MM, Kimura ET and Coltri PP: miR18a and miR19a recruit specific proteins for splicing in thyroid cancer cells. Cancer Genomics Proteomics 14: 373-381, 2017.

11. Small EM, O'Rourke JR, Moresi V, Sutherland LB, McAnally J, Gerard RD, Richardson JA and Olson EN: Regulation of PI3-kinase/Akt signaling by muscle-enriched microRNA-486. Proc Natl Acad Sci USA 107: 4218-4223, 2010.

12. Ren C, Chen H, Han C, Fu D, Zhou L, Jin G, Wang F, Wang D, Chen Y, Ma L, et al: miR-486-5p expression pattern in esophageal squamous cell carcinoma, gastric cancer and its prognostic value. Oncotarget 7: 15840-15853, 2016.

13. Zhu J, Zeng Y, Xu C, Qin H, Lei Z, Shen D, Liu Z and Huang JA: Expression profile analysis of microRNAs and downregulated miR-486-5p and miR-30a-5p in non-small cell lung cancer. Oncol Rep 34: 1779-1786, 2015.

14. Liu C, Li M, Hu Y, Shi N, Yu H, Liu H and Lian H: miR-486-5p attenuates tumor growth and lymphangiogenesis by targeting neuropilin-2 in colorectal carcinoma. Onco Targets Ther 9: 2865-2871, 2016.

15. Cao Z, Liu C, Xu J, You L, Wang C, Lou W, Sun B, Miao Y, Liu X, Wang $X$, et al: Plasma microRNA panels to diagnose pancreatic cancer: Results from a multicenter study. Oncotarget 7: 41575-41583, 2016.

16. Li CY, Liang GY, Yao WZ, Sui J, Shen X, Zhang YQ, Peng H, Hong WW, Ye YC, Zhang ZY, et al: Identification and functional characterization of microRNAs reveal a potential role in gastric cancer progression. Clin Transl Oncol 19: 162-172, 2017.

17. Ma X, Wei J, Zhang L, Deng D, Liu L, Mei X, He X and Tian J: miR-486-5p inhibits cell growth of papillary thyroid carcinoma by targeting fibrillin-1. Biomed Pharmacother 80: 220-226, 2016.

18. Hutter $\mathrm{C}$ and Zenklusen JC: The cancer genome atlas: Creating lasting value beyond its data. Cell 173: 283-285, 2018.

19. Barrett T, Wilhite SE, Ledoux P, Evangelista C, Kim IF, Tomashevsky M, Marshall KA, Phillippy KH, Sherman PM, Holko M, et al: NCBI GEO: Archive for functional genomics data sets-update. Nucleic Acids Res 41 (Database Issue): D991-D995, 2013.

20. Kolesnikov N, Hastings E, Keays M, Melnichuk O, Tang YA, Williams E, Dylag M, Kurbatova N, Brandizi M, Burdett T, et al: ArrayExpress update-simplifying data submissions. Nucleic Acids Res 43 (Database Issue): D1113-D1116, 2015.

21. Chandran UR, Medvedeva OP, Barmada MM, Blood PD, Chakka A, Luthra S, Ferreira A, Wong KF, Lee AV, Zhang Z, et al: TCGA expedition: A data acquisition and management system for TCGA data. PLos One 11: e0165395, 2016.

22. Lassalle S, Zangari J, Popa A, Ilie M, Hofman V, Long E, Patey M, Tissier F, Belléannée $\mathrm{G}$, Trouette $\mathrm{H}$, et al: MicroRNA-375/SEC23A as biomarkers of the in vitro efficacy of vandetanib. Oncotarget 7: 30461-30478, 2016. 
23. Minna E, Romeo P, Dugo M, De Cecco L, Todoerti K, Pilotti S, Perrone F, Seregni E, Agnelli L, Neri A, et al: miR-451a is underexpressed and targets AKT/mTOR pathway in papillary thyroid carcinoma. Oncotarget 7: 12731-12747, 2016.

24. Rossing M, Borup R, Henao R, Winther O, Vikesaa J, Niazi O, Godballe C, Krogdahl A, Glud M, Hjort-Sørensen C, et al: Down-regulation of microRNAs controlling tumourigenic factors in follicular thyroid carcinoma. J Mol Endocrinol 48: 11-23, 2012.

25. Lau J, Ioannidis JP and Schmid CH: Quantitative synthesis in systematic reviews. Ann Intern Med 127: 820-826, 1997.

26. Maragkakis $M$, Vergoulis $T$, Alexiou P, Reczko M, Plomaritou K, Gousis M, Kourtis K, Koziris N, Dalamagas T and Hatzigeorgiou AG: DIANA-microT Web server upgrade supports Fly and Worm miRNA target prediction and bibliographic miRNA to disease association. Nucleic Acids Res 39: W145-W148, 2011.

27. Paraskevopoulou MD, Georgakilas G, Kostoulas N, Vlachos IS Vergoulis T, Reczko M, Filippidis C, Dalamagas T and Hatzigeorgiou AG: DIANA-microT web server v5.0: Service integration into miRNA functional analysis workflows. Nucleic Acids Res 41: W169-W173, 2013.

28. Betel D, Wilson M, Gabow A, Marks DS and Sander C: The microRNA.org resource: Targets and expression. Nucleic Acids Res 36 (Database Issue): D149-D153, 2008

29. Tsang JS, Ebert MS and van Oudenaarden A: Genome-wide dissection of microRNA functions and cotargeting networks using gene set signatures. Mol Cell 38: 140-153, 2010.

30. Wong N and Wang X: miRDB: An online resource for microRNA target prediction and functional annotations. Nucleic Acids Res 43 (Database Issue): D146-D152, 2015.

31. Vejnar CE, Blum M and Zdobnov EM: miRmap web: Comprehensive microRNA target prediction online. Nucleic Acids Res 41: W165-W168, 2013.

32. Hsu SD, Chu CH, Tsou AP, Chen SJ, Chen HC, Hsu PW, Wong YH, Chen YH, Chen GH and Huang HD: miRNAMap 2.0: Genomic maps of microRNAs in metazoan genomes. Nucleic Acids Res 36 (Database Issue): D165-D169, 2008.

33. Blin K, Dieterich C, Wurmus R, Rajewsky N, Landthaler M and Akalin A: DoRiNA 2.0-upgrading the doRiNA database of RNA interactions in post-transcriptional regulation. Nucleic Acids Res 43 (Database Issue): D160-D167, 2015.

34. Weingarten-Gabbay S, Elias-Kirma S, Nir R, Gritsenko AA, Stern-Ginossar N, Yakhini Z, Weinberger A and Segal E: Comparative genetics. Systematic discovery of cap-independent translation sequences in human and viral genomes. Science 351: pii: aad4939, 2016

35. Miranda KC, Huynh T, Tay Y, Ang YS, Tam WL, Thomson AM, Lim B and Rigoutsos I: A pattern-based method for the identification of MicroRNA binding sites and their corresponding heteroduplexes. Cell 126: 1203-1217, 2006.

36. Rehmsmeier M, Steffen P, Hochsmann M and Giegerich R: Fast and effective prediction of microRNA/target duplexes. RNA 10 : $1507-1517,2004$.

37. Cheng Y, Chen L, Cao X, Ha S and Xie X: Expression profiling and functional analysis of hsa-miR-125b and its target genes in drug-resistant cell line of human gastric cancer. Yi Chuan 36: 119-126, 2014 (In Chinese).

38. Pathan M, Keerthikumar S, Ang CS, Gangoda L, Quek CY, Williamson NA, Mouradov D, Sieber OM, Simpson RJ, Salim A, et al: FunRich: An open access standalone functional enrichment and interaction network analysis tool. Proteomics 15: 2597-2601, 2015.

39. Huang da W, Sherman BT and Lempicki RA: Bioinformatics enrichment tools: Paths toward the comprehensive functional analysis of large gene lists. Nucleic Acids Res 37: 1-13, 2009.

40. Szklarczyk D, Morris JH, Cook H, Kuhn M, Wyder S, Simonovic M, Santos A, Doncheva NT, Roth A,Bork P, et al: The STRING database in 2017: Quality-controlled protein-protein association networks, made broadly accessible. Nucleic Acids Res 45: D362-D368, 2017.

41. Gao J, Aksoy BA, Dogrusoz U, Dresdner G, Gross B, Sumer SO, Sun Y, Jacobsen A, Sinha R, Larsson E, et al: Integrative analysis of complex cancer genomics and clinical profiles using the cBioPortal. Sci Signal 6: pl1, 2013.

42. Uhlen M, Fagerberg L, Hallstrom BM, Lindskog C, Oksvold P, Mardinoglu A, Sivertsson Å, Kampf C, Sjöstedt E, Asplund A, et al: Proteomics. Tissue-based map of the human proteome. Science 347: 1260419, 2015.

43. Li W, Wang Y, Zhang Q, Tang L, Liu X, Dai Y, Xiao L, Huang S, Chen L, Guo Z, et al: MicroRNA-486 as a biomarker for early diagnosis and recurrence of non-small cell lung cancer. PLos One 10: e0134220, 2015.
44. He M, Wang G, Jiang L, Qiu C, Li B, Wang J and Fu Y: miR-486 suppresses the development of osteosarcoma by regulating PKC- $\delta$ pathway. Int J Oncol 50: 1590-1600, 2017.

45. Zhang G, Liu Z, Cui G, Wang X and Yang Z: MicroRNA-486-5p targeting PIM-1 suppresses cell proliferation in breast cancer cells. Tumour Biol 35: 11137-11145, 2014.

46. Borjigin N, Ohno S, Wu W, Tanaka M, Suzuki R, Fujita K, Takanashi M, Oikawa K, Goto T, Motoi T, et al: TLS-CHOP represses miR-486 expression, inducing upregulation of a metastasis regulator PAI-1 in human myxoid liposarcoma. Biochem Biophys Res Commun 427: 355-360, 2012.

47. Mees ST, Mardin WA, Sielker S, Willscher E, Senninger N, Schleicher C, Colombo-Benkmann M and Haier J: Involvement of CD40 targeting miR-224 and miR-486 on the progression of pancreatic ductal adenocarcinomas. Ann Surg Oncol 16: 2339-2350, 2009

48. Wang LS, Li L, Li L, Chu S, Shiang KD, Li M, Sun HY, Xu J, Xiao FJ, Sun G, et al: MicroRNA-486 regulates normal erythropoiesis and enhances growth and modulates drug response in CML progenitors. Blood 125: 1302-1313, 2015.

49. Shaham L, Vendramini E, Ge Y, Goren Y, Birger Y, Tijssen MR, McNulty M, Geron I, Schwartzman O, Goldberg L, et al: MicroRNA-486-5p is an erythroid oncomiR of the myeloid leukemias of Down syndrome. Blood 125: 1292-1301, 2015.

50. Ma X, Wei J, Zhang L, Deng D, Liu L, Mei X, He X and Tian J: miR-486-5p inhibits cell growth of papillary thyroid carcinoma by targeting fibrillin-1. Biomed Pharmacother 80: 220-226, 2016.

51. Borzi C, Calzolari L, Centonze G, Milione M, Sozzi G and Fortunato O: mir-660-p53-mir-486 Network: A new key regulatory pathway in lung tumorigenesis. Int J Mol Sci 18: pii: E222, 2017.

52. Oh HK, Tan AL, Das K, Ooi CH, Deng NT, Tan IB, Beillard E, Lee J, Ramnarayanan K, Rha SY, et al: Genomic loss of miR-486 regulates tumor progression and the OLFM4 antiapoptotic factor in gastric cancer. Clin Cancer Res 17: 2657-2667, 2011.

53. Sun H, Cui C, Xiao F, Wang H, Xu J, Shi X, Yang Y, Zhang Q, Zheng $\mathrm{X}$, Yang $\mathrm{X}$, et al: miR-486 regulates metastasis and chemosensitivity in hepatocellular carcinoma by targeting CLDN10 and CITRON. Hepatol Res 45: 1312-1322, 2015.

54. Frietsch JJ, Kastner C, Grunewald TG, Schweigel H, Nollau P, Ziermann J, Clement JH, La Rosée P, Hochhaus A and Butt E: LASP1 is a novel BCR-ABL substrate and a phosphorylation-dependent binding partner of CRKL in chronic myeloid leukemia. Oncotarget 5: 5257-5271, 2014.

55. Yang X, Lv W, Shi R, Cheng S, Zhang J and Xu Z: The clinical implications of Crk-like adaptor protein expression in papillary thyroid microcarcinoma. Tumour Biol 35: 12435-12440, 2014.

56. Zhang J, Chen D, Liang S, Wang J, Liu C, Nie C, Shan Z, Wang L, Fan Q and Wang F: miR-106b promotes cell invasion and metastasis via PTEN mediated EMT in ESCC. Oncol Lett 15: 4619-4626, 2018.

57. Gao ZJ, Yuan WD, Yuan JQ, Yuan K and Wang Y: miR-486-5p functions as an oncogene by targeting PTEN in non-small cell lung cancer. Pathol Res Pract 214: 700-705, 2018

58. Wu S, Wang J and Li F: Dysregulation of PTEN caused by the underexpression of microRNA130b is associated with the severity of lupus nephritis. Mol Med Rep 17: 7966-7972, 2018.

59. Fang Z, Song R, Gong C, Zhang X, Ren G, Li J, Chen Y, Qiu L, Mei L, Zhang R, et al: Ribonucleotide reductase large subunit M1 plays a different role in the invasion and metastasis of papillary thyroid carcinoma and undifferentiated thyroid carcinoma. Tumour Biol 37: 3515-3526, 2016.

60. Zhao Y, Liu X, Zhong L, He M, Chen S, Wang T and Ma S: The combined use of miRNAs and mRNAs as biomarkers for the diagnosis of papillary thyroid carcinoma. Int J Mol Med 36: 1097-1103, 2015.

61. Geeves MA, Hitchcock-DeGregori SE and Gunning PW: A systematic nomenclature for mammalian tropomyosin isoforms. J Muscle Res Cell Motil 36: 147-153, 2015.

62. Bastos AU, de Jesus AC and Cerutti JM: ETV6-NTRK3 and STRN-ALK kinases fusions are recurrent events in papillary thyroid cancer of adult population. Eur J Endocrinol 178: 85-93, 2018.

63. Iyama K, Matsuse M, Mitsutake N, Rogounovitch T, Saenko V, Suzuki K, Ashizawa M, Ookouchi C, Suzuki S, Mizunuma H, et al: Identification of three novel fusion oncogenes, SQSTM1/NTRK3, AFAP1L2/RET, and PPFIBP2/RET, in thyroid cancers of young patients in fukushima. Thyroid 27: 811-818, 2017.

This work is licensed under a Creative Commons Attribution-NonCommercial-NoDerivatives 4.0 International (CC BY-NC-ND 4.0) License. 